\title{
Cutis Verticis Gyrata (CVG)-Radiological Correlation
}

\author{
Dinesh $\mathrm{KC}^{1 *}$ and Meenakshi ${ }^{2}$ \\ ${ }^{1}$ Consultant Radiologist, Shah Satnam ji Speciality Hospitals, India \\ ${ }^{2}$ Consultant Medicine, Shah Satnam ji Speciality Hospitals, India
}

*Corresponding author: Dinesh Kumar Chauhan, Consultant Radiologist, Shah

Satnam ji Speciality Hospitals, Near Shah Satnam Ji Dham, Sirsa, Haryana-125055, India, Tel: 0166-260223; Email: drchauhandinesh@gmail.com

\section{Abstract}

Cutis verticis gyrata (CVG) is a morphological condition characterized with proliferation and thickening of scalp skin which result in deep furrows and convolutions with cerebriform appearance. Typically the skin folds in scalp are seen in an anteroposterior direction, though sometimes they may be seen in a horizontal direction or less commonly in irregular manner.

Keywords: Cutis verticis gyrate; Skin folds; Primary and secondary

Abbreviations: CVG: Cutis Verticis Gyrata; CT: Computed Tomography; MRI: Magnetic Resonance Imaging; 3D VR: Three Dimensional Volumes Rendering.

\section{Introduction}

CVG is rare entity with estimated prevalence of 1 in 100,000 and is more common in males than females ( 5 to 6:1) [1-3]. CVG was classified into primary and secondary form by Polan and Butterworth in 1953 and Garden and Robinson further improved the classification by dividing primary form into primary essential and primary nonessential. The primary essential form usually starts during or after puberty, usually after 30 years of age and show only thick scalp skin folds [2]. No other associated comorbidities are seen with primary essential forms whereas nonessential primary form may show associated neurological or ophthalmological manifestations. Neurological manifestations can be mental retardation, cerebral palsy, microcephaly or seizures. The ophthalmological changes may include cataract, strabismus, blindness, retinitis pigmentosa [4].

\section{Case Report}

A 17 year old male patient presented to outpatient department in our hospital with complaints of increased bulk and thickening of scalp skin for last four to five years, no other cutaneous complaints were present. Patient denies any other associated neurological, ophthalmological complaints or similar family history. No past history of anabolic steroid or any other drug abuse, diabetes mellitus or any other systemic illness was given by patient. Computed tomography and magnetic resonance imaging (MRI) of the patient showed thick, convoluted skin folds giving a cerebriform pattern appearance. Skin fold were seen in typical anteroposterior direction on sagittal view in vertex and in horizontal direction in occipital region on three dimensional volumes rendering (3D VR) computed tomography images. Pituitary fossa was normal, no e/o any pituitary macroadenoma seen on imaging. There were several large skin folds in the sagittal axis on the vertex region, and in the coronal axis on the occipital region (Figures 1-4). 


\section{Clinical Radiology \& Imaging Journal}
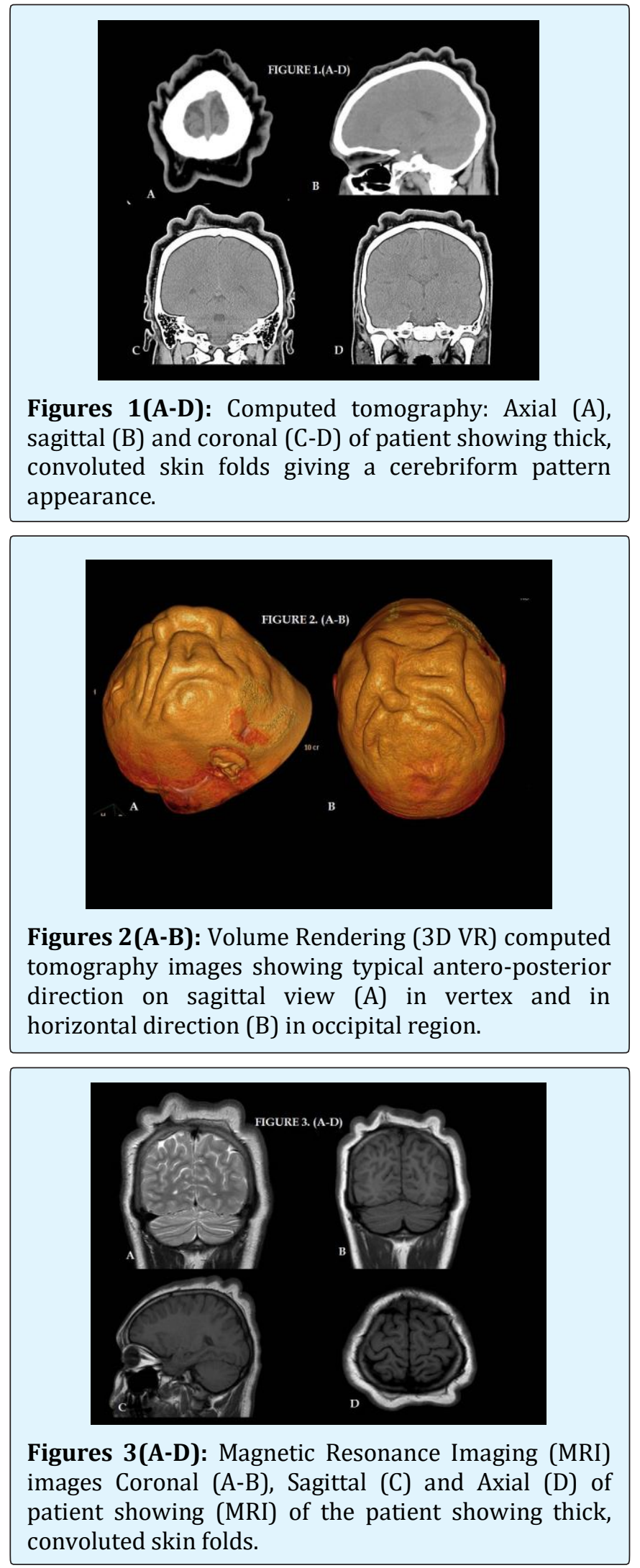

Dinesh KC and Meenakshi. Cutis Verticis Gyrata (CVG)-Radiological Correlation. Clin Radiol Imaging J 2019, 3(4): 000157.

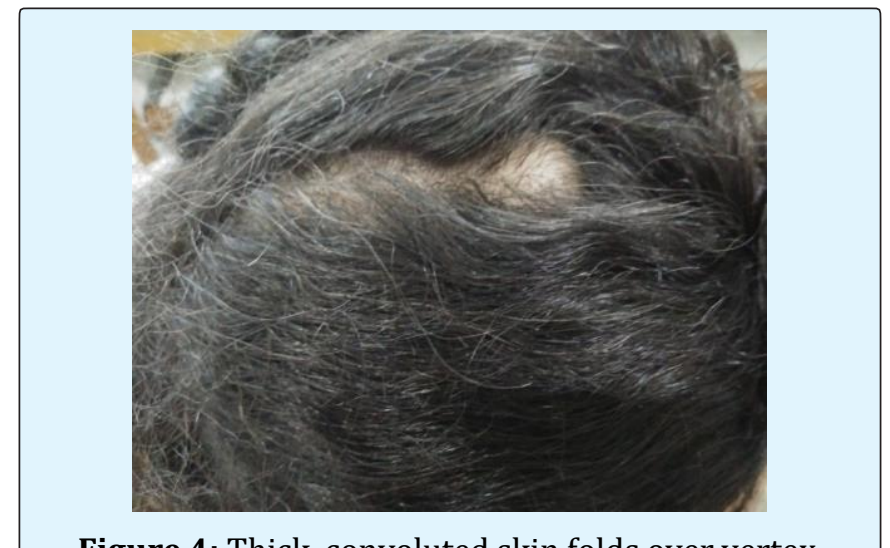

Figure 4: Thick, convoluted skin folds over vertex.

\section{Discussion}

CVG was first described by Jean-Louis-Marc Alibert in 1837and Cutis Verticis Gyrata being proposed by Unna in 1907 [4,5]. In 1953 Polan and Butter-worth in their review of 195 cases, classified CVG as primary (47.7\%) and as secondary (52.3\%). The main associations with secondary form were with tumors/nevi/fibromata (17\%), inflammatory conditions (approximately 10\%) and acromegaly (14\%) [4,6]. The exact etiology and pathogenesis of CVG remains unknown, few sporadic cases have shown autosomal recessive and dominant factors in the primary essential form and endocrine disturbances in the primary nonessential form.

In secondary CVG pathogenesis will depend on the underlying disease. CVG in its primary form develop before puberty in the majority of cases and only $10 \%$ develop after 30 years of age whereas secondary CVG can appear at any age, depending on the underlying pathology $[2,6]$. Secondary cutis verticis gyrata has been associated with various other conditions, including inflammatory or neoplastic processes, use of anabolic steroids, pachydermoperiostosis, acromegaly, myxedema, cretinism, amyloidosis, idiopathic hypertrophic osteopathy, acanthosis nigricans, syphilis, leukemia, intracerebral aneurysm, tuberous sclerosis, neurofibromatosis, Noonan syndrome, Ehlers-Danlos syndrome, trauma and type 2 diabetes mellitus [5].

On radiological imaging $[7,8]$ in CVG, a typical appearance on MRI and 3D volume rendering computed tomography is characterized by scalp undulations due to thickening of the skin and subcutaneous tissues. The average number of skin folds with CVG in one study was six [7], though it may vary from two to as many as twenty folds $[8,9]$ with width varying from 0.75 to 4.0 centimeter 


\section{Clinical Radiology \& Imaging Journal}

and depth from a few millimeters to 1 centimeter [8]. The direction of the folds is usually anterior to posterior in vertex but may be transverse over the occipital scalp or sometime can be irregular [4]. In primary CVG, folds are usually symmetrical and asymmetrical in secondary form [9]. Our present case also showed symptoms during puberty and imaging showed typical symmetrical skin fold with anteroposterior direction in vertex and transverse direction in the occipital region.

The differential diagnosis of primary essential CVG includes the nonessential form, pachydermoperiostosis, acromegaly, intradermal nevus, Cutis laxa, cerebriform intradermal nevus and inflammatory conditions of the scalp [10]. The skin folds in CVG are potential source of infection due to accumulation of secretions and may result in foetid or butyric acid odour [5,9]. As CVG is slowly progressive and essential a benign condition, treatment is usually symptomatic with educating patient regarding hygiene, local care of skin fold to avoid accumulation of secretions and resultant bad odour. The treatment for primary essential CVG may be symptomatic or surgical. Mainstay of treatment is educating patient regarding local hygiene care and to avoid secretion accumulation which lead to unpleasant odour and superadded infections. Surgical treatment is usually for cosmetic reasons as quality of life may be compromised due to unesthetic aspect. Surgical modalities range from simple excisions to tissue expansion and skin grafts for those cases with more extensive scalp involvement [11]. To conclude our present case highlights the benefits of radiological imaging and beauty of 3D VR multidetector computed tomography to show the characteristic pattern of skin folds in scalp in case of CVG.

\section{References}

1. Nguyen NQ (2003) Cutis verticis gyrata. Dermatol Online J 9(4): 32.
2. López V, Montesinos E, Jordá E (2011) Primary nonessential cutis verticis gyrata. Actas Dermosifiliogr 102(6): 475-476.

3. Walia R, Bhansali A (2011) Cutis verticis gyrata. BMJ Case Reports 2011.

4. Diven DG, Tanus T, Raimer SS (1991) Cutis Verticis Gyrata. Int J Dermatol 30(10): 710-712.

5. Yang JJH, Sano DT, Martins SR, Tebcherani AJ, Sanchez APG (2014) Primary essential cutis verticis gyrateCase report. An Bras Dermatol 89(2): 326-328.

6. Hernández JF, Espejo Jiménez LM, Correa Díaz RL, de la Espriella R (2015) Cutis verticis gyrata in adult male institutionalized population in colombia. Case series. Acta Neurol Colomb 31(4): 404-411.

7. Ibrahim AA (2008) Magnetic Resonance Imaging of Cutis Verticis Gyrata. J Computer Assisted Tomography 32(1): 119-123.

8. Okamoto $\mathrm{K}$, Ito J, Tokiguchi S, Ishikawa $\mathrm{K}$, Furusawa $\mathrm{T}$, et al. (2001) MRI in essential primary cutis verticis gyrata. Neuroradiology 43(10): 841-844.

9. Zeljko-Penavić J, Šimić D, Jurišić K, Gunarić A (2016) Cutis Verticis Gyrata - A Case Report. Acta Dermatovenerol Croat 24(2): 140-143.

10. Schenato LK, Gil T, Carvalho LA, Ricachnevsky N, Sanseverino A, et al. (2002) Essential primary cutis verticis gyrata. J Pediatr (Rio J) 78(1): 75-80.

11. Synder MC, Johnson PJ, Hollins RR (2002) Congenital primary cutis verticis gyrata. Plastic Reconstr Surg 110(3): 818-821. 